\title{
AGING IS NOT SENESCENCE: A SHORT COMPUTER DEMONSTRATION AND IMPLICATIONS FOR MEDICAL PRACTICE
}

\author{
Thiago Oliveira Monaco, Paulo Sergio Panse Silveira
}

doi: 10.1590/S1807-59322009000500013

Monaco TO, Silveira PSP. Aging is not senescence: a short computer demonstration and implications for medical practice. Clinics. 2009;64(5):451-7.

INTRODUCTION: The discussion regarding the evolution of aging is almost as old as Darwinian Evolution Theory, but to date, it has remained one of biology's unresolved problems. One issue is how to reconcile natural selection, which is understood as a process that purges deleterious characteristics, with senescence, which seems to offer no advantages to the individual.

METHOD: A computer simulation that illustrates an evolutionary mechanism for the development of senescence in populations is presented.

DISCUSSION: In this article, we debate that two popular explanations for the existence of senescence, namely, (1) the removal of elders for the benefit of the species and (2) the progressive deterioration of the organic machine due to continuous use, are not correct. While human populations continue to age, it is important that the physician understands that senescence, here defined as the progressive impairment of an organism, does not necessarily accompany aging, which we here define as the mere passage of time. As such, we argue that certain processes that were originally assumed to be part of aging should have their status changed because they are actually diseases. Physicians often encounter situations that depend on a better understanding of what limitations senescence imposes on most living species. The concepts of aging (the unavoidable passage of time), senescence (progressive physiologic impairment), and senility (the pathological development of diseases), are discussed.

KEYWORDS: Evolution; Senility; Cellular automata; Mutation accumulation; Computer simulation.

\section{INTRODUCTION}

Medical practice increasingly deals with elderly patients, whose bodies exhibit decreased functional reserves. Indeed, progressive loss of function is a clinical hallmark of aging. Thus, it is a reasonable assumption for physicians that the senile process is a consequence of the exhaustion of the body's mechanisms. Another common idea is that aging is a benefit for the population, resulting in the removal of elderly individuals in favor of young ones. In this paper, we argue that these are misconceptions. Before we can explore possible mechanisms of senescence, we have to distinguish

Discipline of Medical Informatics (LIM-01), Department of Pathology, Faculdade de Medicina da Universidade de São Paulo - São Paulo/SP, Brazil.

Email: thiagomonaco@usp.br

Tel.: 55113064.2744

Received for publication on December 16, 2008

Accepted for publication on February 02, 2009 the concepts of aging, which is the mere passage of time, from senescence, which is the decreasing functionality of an organism. We also define senility, which is the set of pathological processes associated with age. In this context, we argue that simultaneous observations of aging and senescence or senility can be assumed to be coincidental.

The concept of individual exhaustion of the body's mechanisms is not supported because man-made machines are not comparable to biological organisms. Biological organisms self-repair, and a perfect self-repairing mechanism would guarantee aging without senescence. If aging without senescence were impossible and the exhaustion of an organism were unavoidable, then there would be no exceptions within the animal kingdom. However, the Blanding's turtle (Emydoidea blandingii) in captivity has shown decreasing mortality and increasing fertility with aging, as if this species could live in a permanent state of young adulthood. However, this turtle is not immortal, since 
it faces famine, predation, and diseases in nature. ${ }^{1,2}$ It may thus be argued that the organic self-repairing mechanisms of this turtle seem to be permanently functional, while for most other species, perhaps senescence depends not on the progressive exhaustion of body mechanisms but on a programmed failure of repair processes. In fact, evidence for a genetic basis and for the transmission of senescence has accumulated in the literature for decades. ${ }^{3,4}$ Our question is: what is the advantage of defective self-repair for natural selection? We seek to identify an advantage that could function at the population level.

The explanation of senescence as a populational mechanism of sacrificing elderly individuals to benefit the whole species was one of the oldest evolutionary ideas, attempting to reconcile Darwinian evolution and senescence as proposed by August Weismann in $1881 .{ }^{5}$ Weismann, however, could not find a Darwinian mechanism for his proposition. His proposition involved a circular argument since it started with the distinction between younger and older individuals in order to explain the origins of the senile process. In addition, animals of species that do not achieve more advanced ages due to predation can exhibit multiple senile phenomena if they are protected in captivity and allowed to live well past their natural average, even though they may have had no opportunity to expose these traits to natural selection in the course of their evolutionary histories. For those reasons, Weismann himself withdrew his hypothesis a few years later, ${ }^{6}$ but his concept is still repeated today in non-specialized literature.

What, then, is senescence? How can we solve the paradox, since it is widely known that Darwinian natural selection purges deleterious features from organisms, and the senile process is clearly non-defensible as some kind of individual improvement? Senescence is defined by increasing mortality and decreasing fertility with increasing age. In humans, the probability of death is typically higher during the first years of life, progressively decreases until the beginning of the reproductive period, and then steadily increases through advanced ages (Figure 1). Fertility has the opposite behavior; it is null until reproductive age, quickly achieves a peak, and then progressively decreases, trending to zero at menopause in women (Figure 1). The greater the rate of mortality increase (or fertility decrease), the quicker are the senescence processes of a species. These processes seem to be genetically conditioned; the progressive frailty associated with senescence is related to an increasing risk of death that has been shown to double over regular time intervals, the so-called mortality rate doubling time (MRDT), as defined by Gompertz. ${ }^{7}$ The MRDT is a species-specific, empirical measurement of the rate of senescence. MRDT is about 0.3 years in mice, 4.1 years in baboons, and 8.3 years in humans. ${ }^{8}$ Mechanisms that interfere with the rate of senescence, therefore, must change the MRDT. Identifying processes that change the MRDT requires locating the mechanisms that are responsible for senescence.

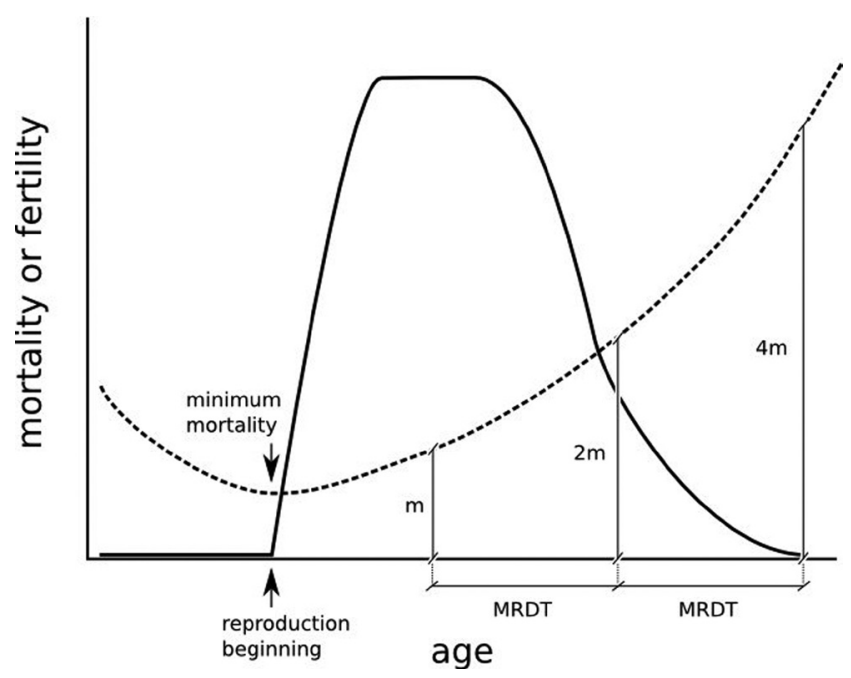

Figure 1 - Hypothetical mortality (dashed line) and fertility (solid line). Mortality decreases from birth to a minimum and then slowly increases with age. In this ascending phase, the probability of death (m) doubles at fixed intervals, which is known as the mortality rate doubling time (MRDT). The beginning of reproduction typically coincides with the minimum mortality, quickly achieves a plateau, and then gradually decreases. Those phenomena are characteristic of a senescent species.

The first competitive evolutionary theory for aging was proposed in 1952 by Medawar and is known as the mutation accumulation theory. ${ }^{9}$ Medawar described a hypothetical population subject to a constant risk of death, which may correspond to predation, starvation, and diseases. This population ages in the sense of passage of time, but it exhibits no senescence in the sense of increased mortality and decreased fertility. The absence of senescence does not imply immortality; instead, due to the accumulated risk of death, this population has far more young individuals than older ones, whose removal would have a negligible impact on general competition for the environment. Medawar therefore proposed that the genetic contribution for the next generation is proportional to the number of individuals that are alive within each age bracket. As such, genes would be more readily exposed to natural selection when expressed in younger individuals, while a deleterious gene could evade natural selection by postponing its expression. This proposition is compatible with Darwinian mechanisms and would cause, over many generations, the accumulation of deleterious mutations and a realignment between the passage of time and senile processes.

In 1957, Williams formalized the antagonistic pleiotropy theory, which proposes that a gene that improves reproduction or survival odds may be favorably selected, 
even if it damages its host later in life. ${ }^{10}$ As such, by the time that the damage is expressed, copies of the genes that cause senescence have already propagated to the next generation. According to Williams' theory, this effect is expected: since most organisms descend from young beings, as Medawar illustrated, the advantage of a minor early benefit that may increase reproductive performance surpasses the disadvantage associated with the much greater damage that may subsequently impair individual robustness.

Although these ideas are not mutually exclusive, difficulties with testing these theories include the multigenerational time scale for evolutionary processes and the paucity of organisms that can be used for experimentation. Therefore, we propose computer simulations. Here we explore Medawar's proposition that natural selection may be inefficient in purging deleterious genes subject to changes in their age of manifestation.

Computational systems that emulate evolutionary rules have been used across different scientific fields, with surprisingly encouraging results. ${ }^{11-14}$ For this study, we developed a computational environment to test the mutation accumulation theory of senescence. Although simple, this model is a demonstration of the progression of a deleterious gene to older ages, as suggested by Medawar. In this context, senescence is interpreted as the summation of many deleterious features that may have been pushed to older ages by Darwinian mechanisms. We argue that understanding this interpretation may be helpful in refining a physician's perspective of disease.

\section{METHODS}

A computer-based approach is an alternative means of testing a theory. It involves simulating a huge number of generations, which would not be feasible given any experimental observation window. For example, the average time of sexual maturation of fruit flies (Drosophila melanogaster) is two weeks. In a simulation where sexual maturation is achieved with forty cycles, the simulation of 800,000 cycles roughly equates to 20,000 generations of fruit flies, corresponding to 766 years of experimentation.

We developed a simple computational model. The environment is represented by a square region that contains digital organisms and simulated food (Figure 2). Each organism is subjected to simple rules defining its movement, feeding, reproduction, and death. These rules are repeatedly applied at every simulation cycle. For instance, the rule for movement changes the position of the individual from its current position to another random position inside a given radius defined by the researchers. Rules for feeding include the environmental distribution of energy units, which each organism can absorb from its surroundings over a given radius. Reproduction depends on contact between males and females of reproductive age. Death features two components: extrinsic mortality, which includes a constant probability of death and starvation due to a lack of energy absorption, and intrinsic mortality, which is the probability of death conditioned by a deleterious gene.

This deleterious gene is the mimicry of the senescent condition. We started all simulations with the deleterious gene expressed at birth. When expressed, the probability of death of its host is the summation of extrinsic and intrinsic mortality. These genes can mutate throughout the simulation. Competing lineages of genes with different ages of manifestation thereby emerge.

\section{Environment}

The environment has periodic boundary conditions, namely, the continuation of the eastern border with the western border and of the northern border with the southern border of the square. This is a condition usually applied to this kind of system to avoid the interference of border effects. Energy units are periodically generated and randomly placed in the environment (Figure 2).

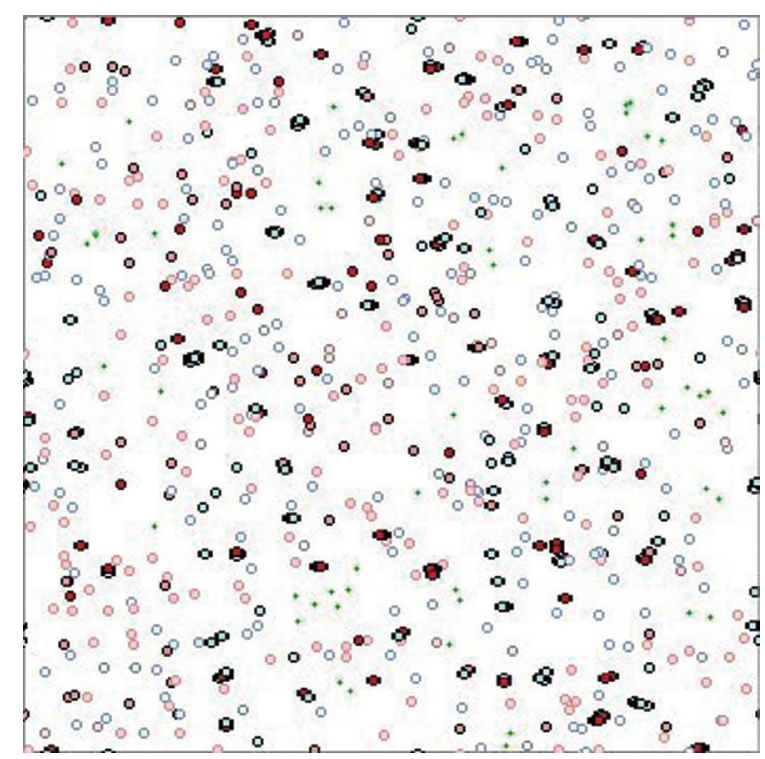

Figure 2 - Visual rendering of the simulated environment. Small dots represent food units, and circles represent individuals. Individuals are depicted in several colors depending on gender, age, and status: blue for males, red for females; pale or intense colors respectively for individuals before and at the onset of reproductive age. Red circles with lateral dots are pregnant females.

\section{Organisms}

Each individual has a collection of variables defining its: - position in the environment; 
- gender (male or female);

- reproductive conditions;

- genotype;

- energy reserve;

- age.

Those variables are changed at every cycle by the rules described above.

\section{Simulations}

The system stores a number of variables to allow the reconstitution of the evolutionary history of each simulation and generates an HTML file to allow visual control by the researcher. We initially conducted a number of preliminary simulations to identify conditions to maintain a stable population. The following conditions were applied to our demonstration:

- Environment: 350 x 350 with periodic boundary conditions;

- 700 units of food per cycle;

- Area for food searching: up to 10 units from an individual's location;

- Individual movement: up to 8 units distant from a current location;

- Area for sexual partner searching: up to 15 units from an individual's location;

- Energy spent per individual: 1 unit per cycle;

- Extrinsic mortality: $0.3 \%$ per cycle;

- Intrinsic mortality (in addition to that associated with gene expression): $1.2 \%$ per cycle;

- Initial age of expression of the death gene: 0;

- Diploid genome, haploid gametes;

- Probability of mutation of the gene: $0.1 \%$ per matching event.

Time passes in discrete cycles. During each cycle, every individual can change its parameters by changing its position, reproducing according to its instantaneous status (too young, at the reproductive age, already pregnant, etc.) by generating gametes that eventually undergo mutations, feeding from the neighborhood, expending its energy reserves, and dying according to intrinsic or extrinsic probabilities of death. It is important to emphasize that all the state changes are local in scope, since the new state depends on the individual's current state and on its neighborhood. The rules for changing state mimic an animal's decision-making behavior, by which we mean that animals only have access to their internal status and to information that is within the reach of their immediate senses.

\section{RESULTS}

The simulated environment has several main ingredients to mimic natural selection: individuals are born, they reproduce with mutations, and they die consistent with a given probability. This probability of death features two components - a constant rate that represents all the environmental risks to which the individual is subjected and an additional probability resulting from the expression of a deleterious gene, which represents senescence in terms of increased fragility of its host.

A typical simulation starts with all diploid individuals hosting a deleterious gene that is expressed at birth (age $=0$ ). Mutations of the deleterious gene occur randomly. At each step of the simulation, the competition between lineages of genes favors the lineage that is accompanied by fewer disadvantages. Our simulation shows the displacement of the average age of manifestation of the deleterious gene from 0 to a plateau near the age of 250 cycles in this example (Figure 3). This behavior is a consequence of the population age structure, which is discussed in the next section.

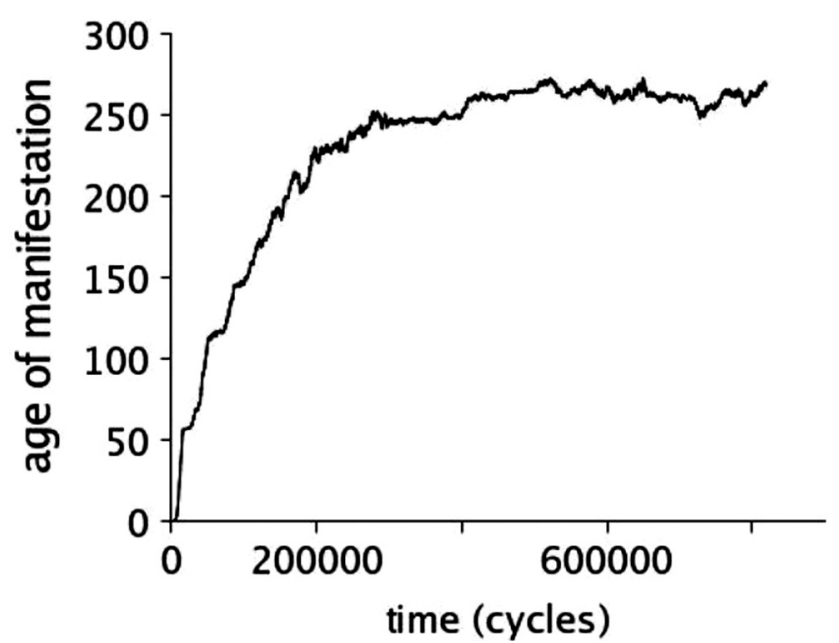

Figure 3 - Typical evolution of the average age of expression of a deleterious gene as a function of the number of simulation steps. At the beginning of a simulation (cycle $=0$ ) the gene expression occurs at birth $($ age $=0)$. Over many generations, genes that express the deleterious effect later are progressively selected. This selection is initially fast, but it decreases with increasing simulation time until it reaches a plateau, which is around 250 cycles (arbitrary scale) in this example.

The age structure that emerges from the properties of our simulations (Figure 4a) is not surprising. In fact, it is comparable to the age-structure of human populations in less developed countries, such as the Afghanistan population in 2008 (Figure 4b). This distribution is also similar to wild, unprotected animal populations, in which birth rates are high, but child mortality quickly reduces the population that reaches reproductive age. 
(a)

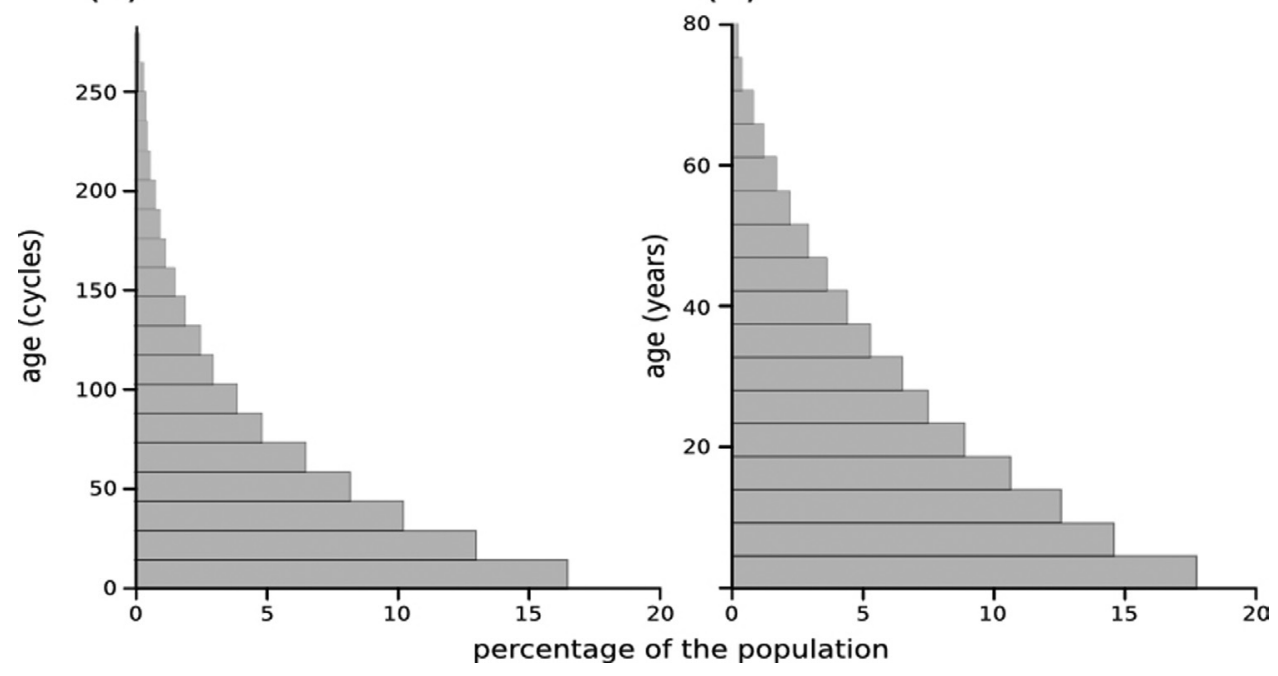

Figure 4 - Age distribution of the populations: (a) typical simulated population showing that very few individuals survive beyond 250 cycles of age, which corresponds to $87 \%$ of the maximum age (compare with figure 3); (b) age distribution of the Afghan population in 2008 showing that very few individuals survive beyond 75 years of age, which corresponds to $89 \%$ of the maximum age (data from U.S. Census Bureau, International Database, available at http:// www.census.gov/).

\section{DISCUSSION}

To try to understand senescence as being some kind of benefit would potentially be misleading. Medawar's proposal is that natural selection can be avoided by genetic strategies such as the mutation of a deleterious gene's age of expression. Under this condition, natural selection is unable to purge a deleterious gene. Instead, the expression of this gene is simply delayed to more advanced ages.

Mutated genes with delayed expression (Figure 3) are selected because the later the expression of a deleterious gene is, the smaller the removal rate of reproductive individuals from the population. Consequently, the smaller is the disturbance associated with leaving descendants to the next generation. In our simulation, very few individuals survive more than 250 cycles (Figure $4 \mathrm{a}$ ). Thus, the facts that the selection process stops at 250 cycles and that the maximum life span is about 250 cycles are not coincidental.

We hypothesize that senescence may be the evolutionary result of many deleterious genes that are accumulated over older ages through this mechanism. This may seemingly link the aging and senility processes. In this paper, we show that this phenomenon of accumulation of deleterious genes at later ages, which is termed "mutation accumulation" in the context of Medawar's theory, can be mimicked in an artificial life system.

Although it is a simple model, postponing the manifestation of a deleterious gene to later ages through random mutation and natural selection is not the only realistic feature demonstrated by our simulation; the rate with which this delay process occurs during the simulation is not constant. The speed of selection is highest at the beginning of the simulation and is virtually null once it reaches the plateau (Figure 3). This rate decrease is a product of the decreasing force of selection, which in turn depends on the number of individuals that are alive within each age bracket. In other words, while mutations of a gene are selected in favor of more advanced ages, at which fewer individuals survive, the force of selection against the deleterious genes progressively decreases with the resulting younger population. A plateau is reached when the number of living individuals hosting the deleterious gene is so small that selection cannot confer an advantage to genes that mutate towards having a deleterious effect at even more advanced ages.

Although present in almost all superior animals and humans, senescence does not seem to be beneficial. To justify the potential benefits of senescence, two fallacious arguments are usually invoked by non-specialists: (a) that selection removes elderly individuals for the welfare of the population or (b) that senescence is an unavoidable process of organic exhaustion analogous to the deterioration of a machine. The first argument fails because the deaths of the few elderly individuals in a relatively young population release a negligible amount of resources to the rest of the population. The second argument sounds plausible but is not supported because of exceptions such as the abovementioned Blanding's turtle. Because organisms, unlike machines, can self-repair, we argue that senescence is not caused by the exhaustion of organic processes but by a programmed failure of the selfrepairing mechanisms. One may theorize about a population 
that is initially free from genes that impair the self-repairing mechanisms and is thus free of senescence. Senescence, by the mechanisms simulated here, would organically develop in a population once a new mutation introduces such a gene. Even if this gene were expressed at birth (as in the case of our simulation), natural selection would displace this gene to older ages. If many other deleterious genes can also accumulate with this mechanism, the net result will be the senile process. Blanding's turtles appear to be an exception in the sense that virtually none of these deleterious genes seem to have appeared along their evolutionary trajectory, while for most of the other species, natural selection impaired the self-repairing mechanisms by pushing deleterious genes toward advanced ages. We often consider aging and senescence as synonymous due to the temporal coincidence in their symptoms, but, in fact, we argue that they are not linked. Being aware of the distinction between aging and senescence has several implications for clinical practice.

We mentioned in the introduction that senescence, the "normal aging" feature of a population, can be described in terms of its MRDT, which is the regular time-interval at which mortality rates ordinarily double. Here we repeat the definition of senescence as a natural process, as opposed to senility, which is the effect of accumulated pathological processes upon the organism. The increase in mortality rates measured by MRDT is species-specific because it is determined by the programmed features of a given species. Senility, or "pathological aging," is characterized instead by organic limitations imposed by an individual's life history and choices, as well as its particular genetic inheritance. Such limitations are neither regular for all age brackets, nor are they speciesspecific, since they are most often associated with diseases.

For instance, creatinine clearance capacity in humans normally starts decreasing as early as the 30 s and continues to decrease, usually reaching critically low levels at ages as advanced as 100 years (Figure 5). Other organs and systems also exhibit similar spontaneous decreases, which are assumed to be a part of normal senescence. Senility, on the other hand, can be elicited by bad life habits or by lack of adherence to treatment regimens for hypertension or diabetes, both of which cause organ function to be severely compromised at ages much younger than 100 years.

It is relevant for clinical practitioners to distinguish senescence and senility when dealing with patients. The effects of natural aging are considered inexorable, and the clinician can only learn to deal with them; as to the effects of pathological aging, we are taught to prevent, treat and rehabilitate as best we can. Nevertheless, the distinction between senescence and senility, though useful, is sometimes difficult even for the experienced geriatrician. In contrast with our computational example, we still cannot understand the genetic basis of our evolution, which demonstrates why it is hard to distinguish senescence from senility.

Given this difficulty, medical history has witnessed changes in the classification of processes in the elderly from pathological to normal and vice versa, as is the case with arterial hypertension in the elderly; once thought to be a normal part of the aging process, it is now considered pathological. ${ }^{15}$

To reconcile such traditional geriatric models with the evolutionary perspective, the MRDT is helpful. Genetic features that change the MRDT are a part of a species' senescence process, while genes related to diseases may only change survival probabilities, without changing the rate of mortality doubling. ${ }^{16}$ In fact, although most genes only impact survival, a small set of genes is capable of changing the MRDT, suggesting that something in the very process of senescence can indeed be delayed. ${ }^{16}$ Such results shed light on the fuzzy boundaries between senescence and senility.

\section{CONCLUSION}

At the time of this writing, most national health promotion strategies focus on the processes of senility. If genes related to repair systems can be manipulated in the future, a second strategy may become a reality - namely, a manipulation of the processes of senescence. The expected effects of such strategies are exemplified in figure 5, which

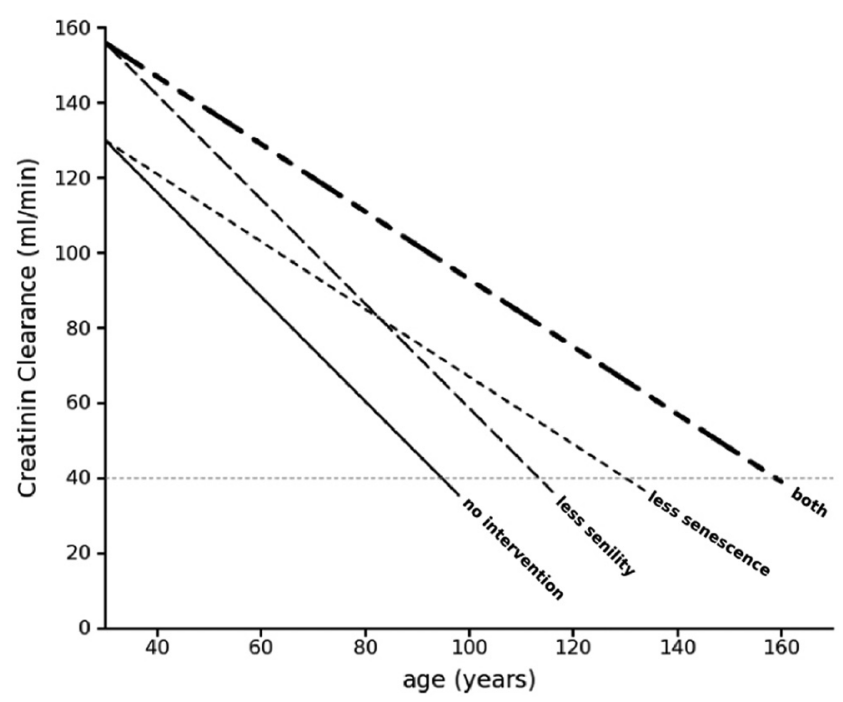

Figure 5 - Creatinine clearance decreases linearly with age. At a normal rate, departing from a normal clearance of $130 \mathrm{ml} / \mathrm{min}$, the onset of renal insufficiency is expected around the age of 95 years (solid line). A hypothetical treatment interfering with senility that could improve the initial clearance rates would shift the curve to the right, thereby postponing the onset of renal insufficiency (thick dashed line). Alternatively, if we could alter the genetic mechanisms of senescence to change the rate of loss of kidney function, the slope of the curve would change, also postponing the onset of renal insufficiency (thin dashed line). The combination of both approaches could extend renal function to much more advanced ages (thickest dashed line). 
shows that strategies against senility would change the total capability of an organ, while strategies against senescence would change the rate and character of functional loss. Although this kind of intervention is not yet a reality, physicians who are aware of these differences will be ready to recognize the role of combined strategies that may in the future become available to medical professionals.

\section{REFERENCES}

1. Hirsch HR. The waste-product theory of aging: waste dilution by cell division. Mech Ageing Dev. 1978;8:51-62.

2. Congdon JD, Nagle RD, Kinney OM, van Loben Sels RC. Hypotheses of aging in a long-lived vertebrate, Blanding's turtle (Emydoidea blandingii). Exp Gerontol. 2001;36:813-27.

3. Kirkwood TB, Austad SN. Why do we age? Nature 2000 Nov 9;408:2338.

4. Finch CE, Pike MC, Witten M. Slow mortality rate accelerations during aging in some animals approximate that of humans. Science. 1990;24;249:902-5.

5. Kirkwood TB, Cremer T. Cytogerontology since 1881: a reappraisal of August Weismann and a review of modern progress. Hum Genet. 1982;60:101-21.

6. Semsei I. On the nature of aging. Mechanisms of Ageing and development. 2000;117:93-108.

7. Gompertz B. On the nature of the function expressive of the law of human mortality and on a new mode of determining life contingencies. Philos Trans Roy Soc London. 1825;115:513-85.

8. de Magalhaes JP. Is mammalian aging genetically controlled? Biogerontology. 2003;4:119-20.
9. Medawar PB. An Unsolved Problem of Biology. 1952. London, Lewis. Ref Type: Generic

10. Williams GC. Pleiotropy, natural selection and the evolution of senescence. Evolution. 1957;11:398-411.

11. Panse Silveira PS, Massad E. Modeling and simulating morphological evolution in an artificial life environment. Comput Biomed Res. 1998;31:1-17.

12. Lenski RE, Ofria C, Pennock RT, Adami C. The evolutionary origin of complex features. Nature. 2003;8;423:139-44.

13. Chow SS, Wilke CO, Ofria C, Lenski RE, Adami C. Adaptive radiation from resource competition in digital organisms. Science. 2004;2;305846 .

14. Hoffmann JP. Darwin and Computational Ecology: How Simple Models of Evolution Help our Search for Better Models of Ecological Systems. 5 A.D. Dec 6; France: Blaise Pascal University; 2005 p. 27-39.

15. Sander GE. High blood pressure in the geriatric population: treatment considerations. Am J Geriatr Cardiol. 2002;11:223-32.

16. de Magalhaes JP, Cabral JA, Magalhaes D. The influence of genes on the aging process of mice: a statistical assessment of the genetics of aging. Genetics. 2005;169:265-74. 
